\title{
Photothermally Responsive Conjugated Polymeric Singlet Oxygen Carrier for Phase Change-Controlled and Sustainable Phototherapy for Hypoxic Tumor
}

\author{
Guo Li, ${ }^{1}$ Ruyi Zhou, ${ }^{1}$ Weili Zhao, ${ }^{1}$ Bo Yu, ${ }^{1}$ Jie $\mathrm{Zhou}^{1}{ }^{1}$ Shujuan Liu, ${ }^{1}$ Wei Huang, ${ }^{1,2}$ \\ and Qiang Zhao' \\ ${ }^{1}$ Key Laboratory for Organic Electronics and Information Displays \& Jiangsu Key Laboratory for Biosensors, Institute of Advanced \\ Materials (IAM), Nanjing University of Posts and Telecommunications (NUPT), 9 Wenyuan Road, Nanjing, 210023 Jiangsu, China \\ ${ }^{2}$ Frontiers Science Center for Flexible Electronics, Xi'an Institute of Flexible Electronics (IFE) and Xi'an Institute of Biomedical \\ Materials \& Engineering, Northwestern Polytechnical University, 127 West Youyi Road, Xi'an 710072, China
}

Correspondence should be addressed to Shujuan Liu; iamsjliu@njupt.edu.cn, Wei Huang; iamdirector@fudan.edu.cn, and Qiang Zhao; iamqzhao@njupt.edu.cn

Received 14 July 2020; Accepted 25 August 2020; Published 10 October 2020

Copyright (C) 2020 Guo Li et al. Exclusive Licensee Science and Technology Review Publishing House. Distributed under a Creative Commons Attribution License (CC BY 4.0).

\begin{abstract}
Hypoxia significantly compromises the therapeutic performance of photodynamic therapy (PDT) owing to the oxygen level which plays a key role in the production of singlet oxygen $\left({ }^{1} \mathrm{O}_{2}\right)$. Herein, the photothermally responsive phase change materials $(\mathrm{PCM})$ are used to encapsulate 1,4-dimethylnaphthalene-functionalized platinum(II)-acetylide conjugated polymer (CP1) with intense nearinfrared (NIR) absorption to prepare new ${ }^{1} \mathrm{O}_{2}$ nanocarriers (CP1-NCs). The 1,4-dimethylnaphthalene moieties in CP1-NCs can trap the ${ }^{1} \mathrm{O}_{2}$ produced from $\mathrm{CP} 1$ under irradiation and form a stable endoperoxide. Then, the endoperoxide undergoes cycloreversion to controllably release ${ }^{1} \mathrm{O}_{2}$ via the NIR light-triggered photothermal effect of $\mathrm{CP} 1$ and controllable phase change of PCM, which can be used for oxygen-independent PDT for hypoxic tumor. Furthermore, the in vivo luminescence imagingguided synergistic PDT and photothermal therapy showed better efficiency in tumor ablation. The smart design shows the potent promise of CP1-NCs in PCM-controlled and sustainable phototherapy under tumor hypoxic microenvironment, providing new insights for constructing oxygen-independent precise cancer phototherapeutic platform.
\end{abstract}

\section{Introduction}

Photodynamic therapy (PDT), as a well-known and emerging clinical treatment solution, can transform oxygen $\left(\mathrm{O}_{2}\right)$ into toxic reactive oxygen species (ROS) to eradicate cancer cells [1-4]. As a consequence, considerable efforts have been devoted to enhancing PDT due to their minimal invasiveness, high selectivity, accurate spatiotemporal regulation, and minimized side effect [5-9]. So far, a large majority of reported PDT therapeutic systems depend highly on $\mathrm{O}_{2}$ level [10-14]. However, the consumption of $\mathrm{O}_{2}$ in PDT process and rapid proliferation of tumor cells aggravate hypoxic microenvironments [15-17]. Thus, tumor hypoxia is traditionally considered the "Achilles' heel" of PDT, which not only accelerates metastasis in many solid tumor cells but also significantly causes therapy resistance and low antitumor efficiency [18-23].
To date, various innovative strategies have been extensively utilized to relieve tumor hypoxia and enhance the therapeutic effect of PDT $[18,20,21]$. One is to integrate PDT with chemotherapy for synergistic therapy [24-26]. However, such chemophototherapeutic prodrugs are complex multicomponent nanosystems, which often lead to the difficulty in the preparation and suffer from the threat of release of drug in normal cells and tissues. Another approach is to directly transport $\mathrm{O}_{2}$ into tumor sites through oxygencarrier nanomaterials, including perfluorocarbon, $\mathrm{CaO}_{2}$, $\mathrm{MnO}_{2}$, carbon nitride, enzymes, or oxygenated hemoglobin [27-30]. The enhanced PDT efficiency could be achieved by the quantitative $\mathrm{O}_{2}$ generation performance. However, in these oxygen self-supplying nanomaterials, there remain many severe challenges, including unexpected side effects and unpredictable toxicity in the complicated physiological 
environment. In addition, many smart phototherapy platforms for in situ generation of hydroxyl radicals within tumors based on the unique tumor microenvironment feature through Fenton reaction have been developed, which possess high therapeutic specificity and low invasiveness [31-36]. However, most of these nanoplatforms are also based on complex multicomponent nanosystems [27-31]. Although many prominent works have been developed, there is an urgent need to construct a new PDT therapeutic system for overcoming the tumor hypoxia and achieving sustainable phototherapy.

Herein, we developed a novel photothermally responsive conjugated polymeric singlet oxygen $\left({ }^{1} \mathrm{O}_{2}\right)$ carrier, achieving phase change-controlled and luminescence imaging-guided sustainable phototherapy for hypoxic tumor (Figure 1). Platinum(II)-acetylide conjugated polymers (CPs) containing boron dipyrromethene (BDP) units with intense nearinfrared (NIR) absorption were chosen as a perfect phototherapeutic platform [37-44], which simultaneously possess PDT and photothermal therapy (PTT) properties. 1,4Dimethylnaphthalene, as the efficient ${ }^{1} \mathrm{O}_{2}$ carrier, was introduced into CPs (CP1), which can reversibly trap and photothermally release ${ }^{1} \mathrm{O}_{2}$ (Figure $1(\mathrm{a})$ ), providing a new strategy for directly delivering ${ }^{1} \mathrm{O}_{2}$ to relieve hypoxic tumor [45-47]. As compared to many other stimulus-responsive platform, photothermally responsive nanoplatform via phase change materials (PCM) with a large potential fusion heat and reversible solid-liquid phase change in a relative narrow temperature region is more appropriate for accurately controlled release [48-51]. Therefore, the CP1 nanocarriers (CP1-NCs) were prepared through coencapsulating hydrophobic CP1 in biocompatible organic PCM (a mixture of oleic acid and hexadecanol, melting point at $46^{\circ} \mathrm{C}$ ) with amphiphilic lecithin and DSPE-mPEG ${ }_{5000}$. Meanwhile, the novel CP1-NCs were injected into a tumor-bearing mouse via the tail vein and effectively accumulated in tumor location via the improved permeability and retention (EPR) effect [52, 53]. Besides, the CP1-NCs could not only act as a NIR luminescence imaging contrast agent but also trap the ${ }^{1} \mathrm{O}_{2}$ produced from CP1 under irradiation and form a stable endoperoxide (EPO). Furthermore, the EPO undergoes cycloreversion to controllably release ${ }^{1} \mathrm{O}_{2}$ via the NIR light-induced photothermal effect of CP1 and controlled phase change of PCM, which can be used for oxygen-independent PDT for hypoxic tumor. Therefore, the new CP1-NC therapeutic nanoplatform could successfully relieve tumor hypoxia for oxygenindependent sustainable phototherapy and provide valuable guideline to develop high-performance theranostic agents in cancer treatment.

\section{Results and Discussion}

2.1. Synthesis and Characterization. BDP-based CPs (CP1CP3) were synthesized from the BDP precursor and Pt1 or 1,4-diiodobenzene through dehydrohalogenation reaction $[54,55]$. The monomer trans-dichlorobis (tri-n-butylphosphine) platinum(II) (Pt1) was obtained from the reported methods [56-58]. The detailed synthetic routes of BDP precursor and CP1-CP3 are shown in Figures S1 and S2. CP2 and CP3 without Pt center and 1,4-dimethylnaphthalenefunctionalized group were designed as the control. CP1CP3 were purified through silica column chromatography and precipitated in methanol, generating the dark green and purple polymers. The ${ }^{1} \mathrm{H}$ NMR, ${ }^{13} \mathrm{C} \mathrm{NMR}$, and matrixassisted laser desorption/ionization time-of-flight mass spectrometry were used to characterize the purity of intermediates and CPs. Furthermore, the weight average molecular mass $\left(M_{\mathrm{w}}\right)$ of $\mathrm{CP} 1, \mathrm{CP} 2$, and CP3 was 9500, 8500 , and 8500 with polydispersity indexes (PDI) of 1.11 , 1.09 , and 1.14 , respectively.

As shown in Figure 1(b), the CP1-NC formation was illustrated. The PCM were prepared according to the reported procedures [48]. Through modulating the mass ratio of oleic acid (OA) and 1-hexadecanol (Hex) at $1: 3.5$, PCM with a melting point of $46^{\circ} \mathrm{C}$ were obtained (Figure 2(a)). Through coencapsulating hydrophobic CP1CP3 into PCM with amphiphilic lecithin and DSPE$\mathrm{mPEG}_{5000}$, the water-soluble CP1-NC and CP nanoparticles (CP-NPs) were acquired. The concentrations of CP1-NCs and CP-NPs were calculated according to the absorption of Figure S4. The CP1-NCs were fully characterized by transmission electron microscopy (TEM) and dynamic light scattering (DLS). TEM imaging showed that CP1-NCs possessed a uniform morphology, revealing the diameter about $35 \mathrm{~nm}$ (Figure 3(a)). DLS indicated that CP1-NCs possessed an average hydrodynamic size of around $78 \mathrm{~nm}$ (Figure 3(b)), suggesting the potential passive tumor targeting ability via EPR effect [52].

2.2. Photophysical Properties. The photophysical properties of CPs, CP1-NCs, and CP-NPs were explored by the absorption and photoluminescence (PL) spectra. As exhibited in Figures 3(c) and S5, the maximal absorption peaks of CP1-CP3 were located at 660,575, and $650 \mathrm{~nm}$ in $\mathrm{CH}_{2} \mathrm{Cl}_{2}$, respectively, which are assigned to the $\pi-\pi *$ transition of the BDP platinum-acetylide backbone, showing significant red shift in comparison with that of their corresponding BDP precursor owing to the $\pi$-electron delocalization and rigid planar structures $[42,59]$. The absorption spectra of CP1-NCs displayed a strong absorption band at $254 \mathrm{~nm}$, which was consistent with the absorption of CP1 and 6. After the coordination with BDP precursor 6 or 7, CP1-CP3 possessed intense NIR emission in the region of $650-750 \mathrm{~nm}$ (Figure S6). Compared with the CPs, the formed CP-NPs showed significant suppression of the luminescence, which endows a high potential for CP-NPs as a photothermal agent $[38,60]$. The strong $\pi-\pi$ stacking in the micellar cores leads to the quenching of fluorescence [60]. Furthermore, photostability is the key in cancer phototherapy, and the resistance of CP1-NCs to photobleaching was measured. The absorption of CP1NCs showed no significant change during $60 \mathrm{~min}$ under $690 \mathrm{~nm}$ light irradiation, demonstrating the excellent antiphotobleaching performance (Figure 3(d)).

2.3. Photodynamic and Photothermal Properties. The photothermal effect of CP1-NCs plays an important role in the process of triggering ${ }^{1} \mathrm{O}_{2}$ release. Therefore, the temperature 

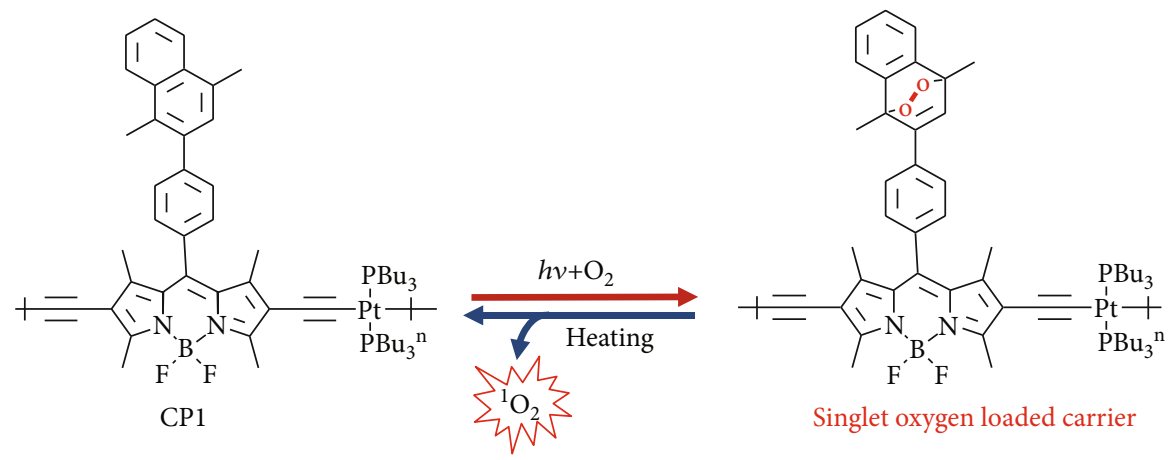

(a)
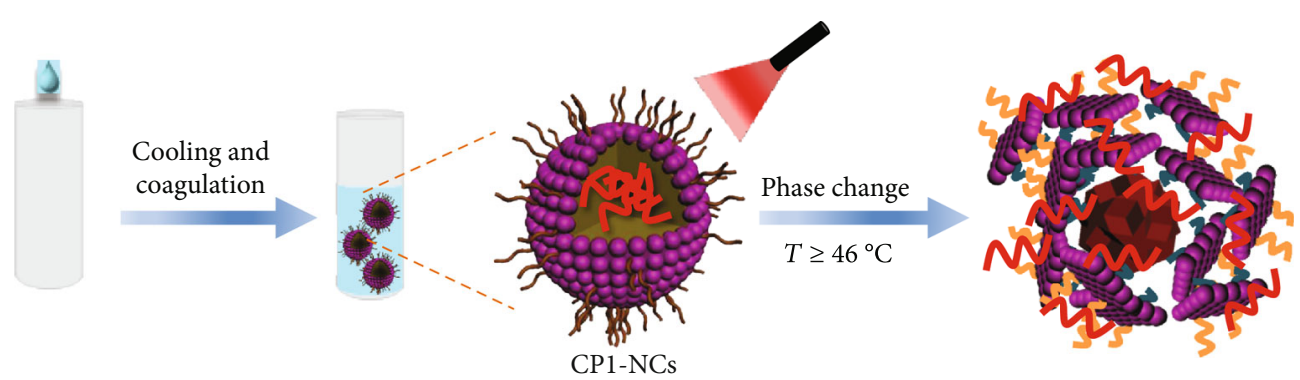

(b)
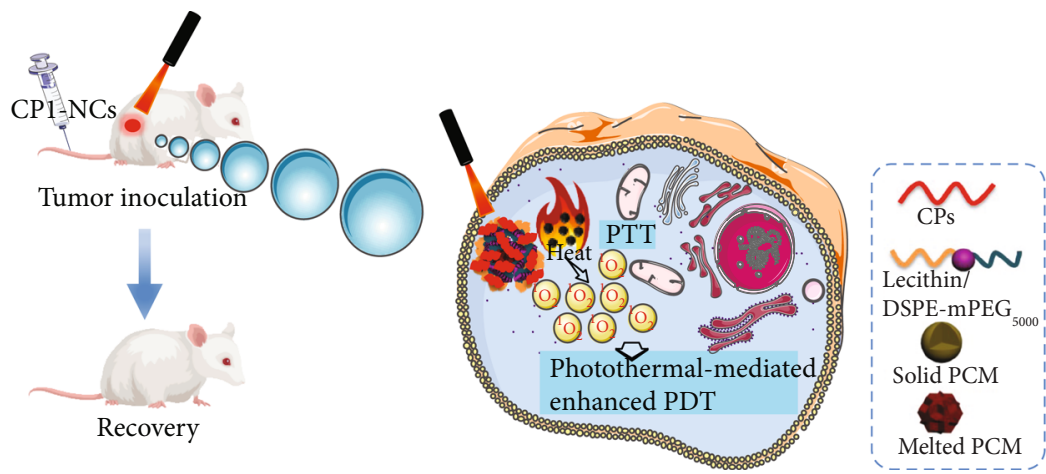

(c)

Figure 1: (a) Mechanism illustration of capture and release of ${ }^{1} \mathrm{O}_{2}$ by the $\mathrm{CP} 1$ as ${ }^{1} \mathrm{O}_{2}$ carrier. (b) The CP1-NC construction through the modified nanoprecipitation and the photothermal-triggered release of CP1-NCs and PCM. (c) The responsive process of controlled and sustainable phototherapy under hypoxia.

elevation of CP1-NCs was firstly explored at various concentrations under $690 \mathrm{~nm}$ irradiation $\left(0.5 \mathrm{~W} \mathrm{~cm}^{-2}\right)$. CP1-NCs showed remarkable temperature elevation from 21 to $39^{\circ} \mathrm{C}$ with the increasing of concentration, which was much higher than that of the control CP2-NPs $\left(\Delta T \approx 9^{\circ} \mathrm{C}\right.$ ) (Figures $2(\mathrm{c})$ and S8). The remarkable temperature elevation of CP1-NCs demonstrates that the introduction of $\mathrm{Pt}$ can enhance the photothermal efficiency through the improved nonradiative decay process [38]. Besides, the photothermal conversion effect of CP1-NCs was tested by temperature elevation through a cycle of heat-up and cooling after cessation of light irradiation. CP1-NCs showed the superior photothermal conversion effect of $49.0 \%$ compared with the existing photothermal materials such as aza-boron-dipyrromethene dye nanoparticles (43.0\%), Pt-based nanoparticles (37.0\%), semiconducting polymer nanoparticles $(26.7 \%)$, cyanine dye nanocarriers $(26.6 \%)$, and gold rods $(21.0 \%)$ [38, 61-64]. Moreover, CP1-NCs exhibited almost no large difference in their temperature monitoring after five irradiation/cooling cycles (Figure S8(a)), suggesting their superior resistance to photobleaching under irradiation.

Then, we explored the morphological changes of the CPNCs before and after $690 \mathrm{~nm}$ laser irradiation. TEM imaging suggested that light irradiation generated significant influence not only on the particle size but also on the morphology of the CP1-NCs@PCM (Figures 2(d) and 2(e)). After irradiation, the morphological change of CP1-NCs was observed from uniform sphere to amorphous structure by TEM imaging. And the hydrodynamic diameters of the nanocarriers became smaller (from $74 \pm 6 \mathrm{~nm}$ to $36 \pm 5 \mathrm{~nm}$ ) through DLS (Figure S3). For CP2-NPs, no significant difference was found in the particle size and morphology of the CP2NPs@PCM after irradiation, which was attributed to the negligible photothermal efficiency of CP2-NPs.

Afterwards, the sustainable PDT property of CP1-NCs was demonstrated through investigating the ${ }^{1} \mathrm{O}_{2}$ trap and 


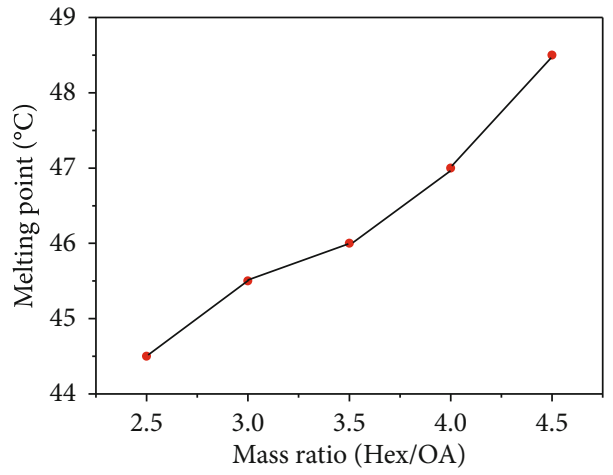

(a)

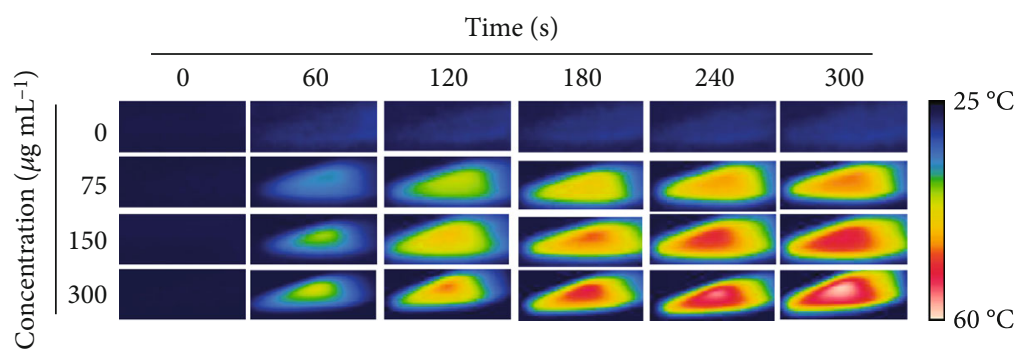

(b)

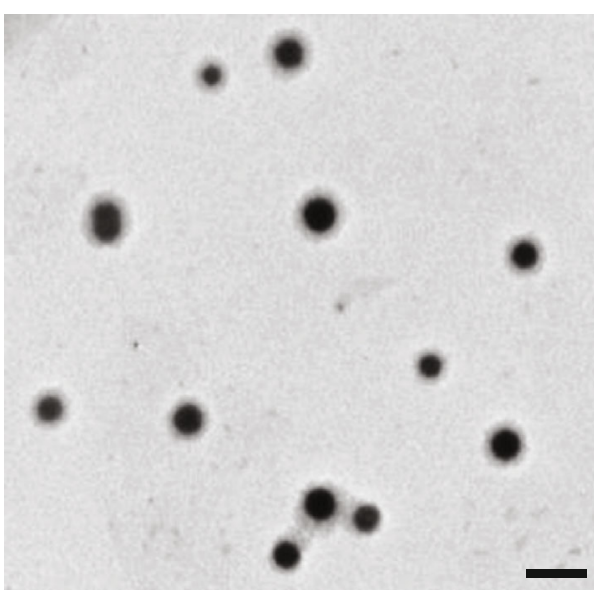

(d)
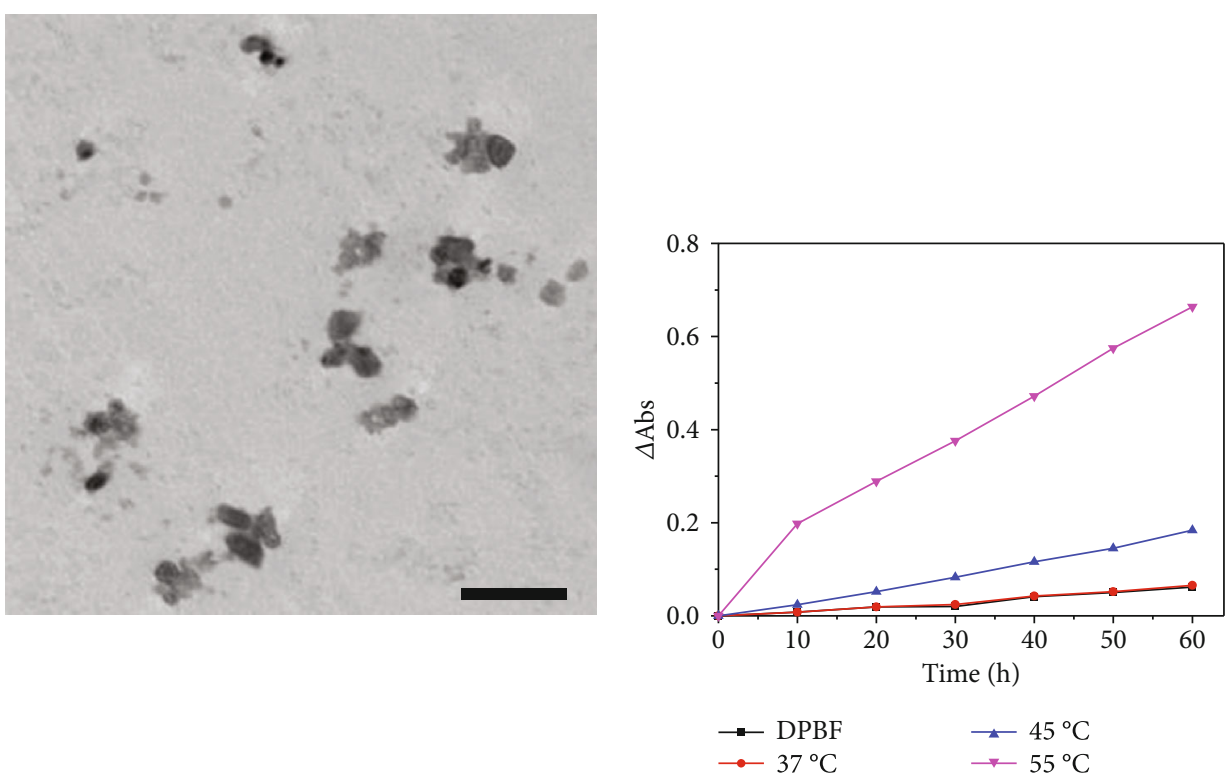

(f)

Figure 2: Continued. 


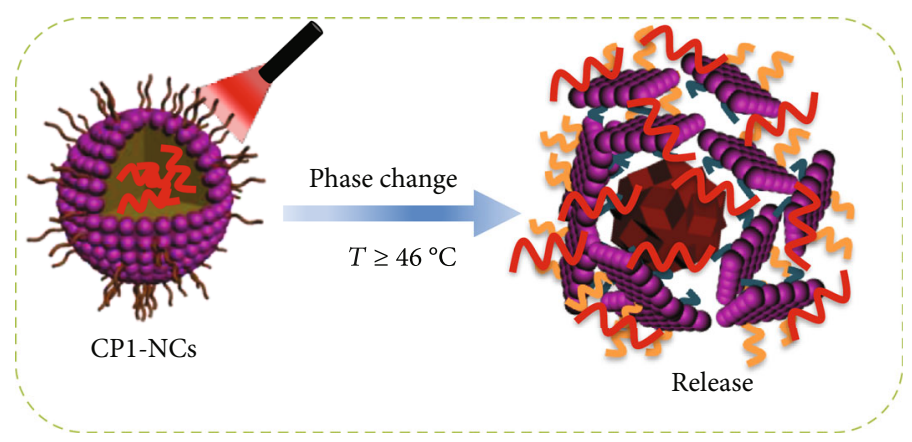

(g)

Figure 2: (a) Melting point change of PCM with different Hex/OA mass ratios. (b) Photothermal images of CP1-NCs. (c) Temperature elevation of CP1-NCs at different levels under $690 \mathrm{~nm}$ light irradiation at $0.5 \mathrm{~W} \mathrm{~cm}^{-2}$ for $6 \mathrm{~min}$. TEM of CP1-NCs, (d) before, and (e) after irradiation for $6 \mathrm{~min}$. Scale bars: $200 \mathrm{~nm}$. (f) $\triangle \mathrm{Abs}$ of DPBF under various temperatures in the mixture solution of CP1-NCs and DBPF. (g) Schematic illustration of photothermal-induced release of CP1-NCs.

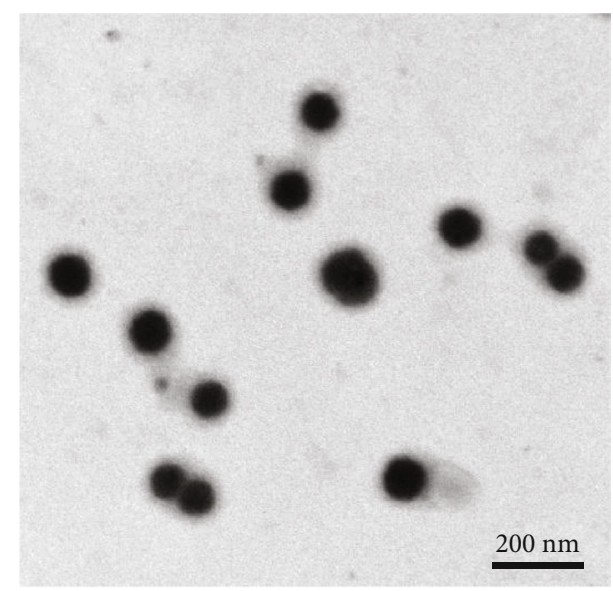

(a)

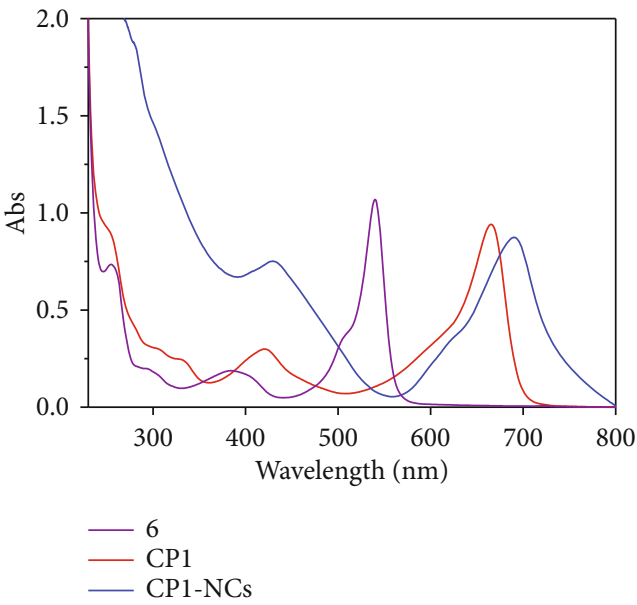

(c)

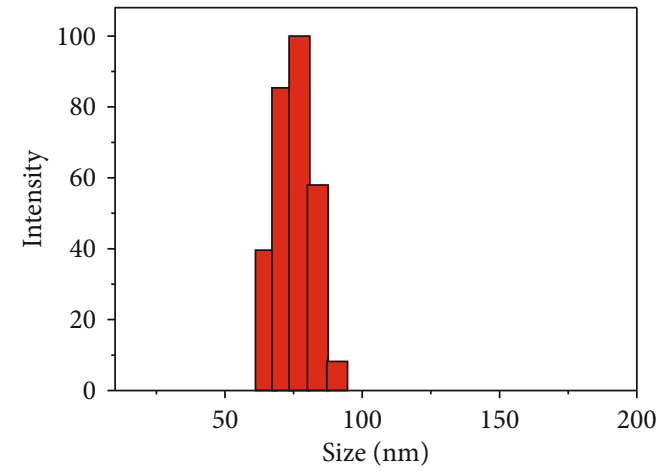

(b)

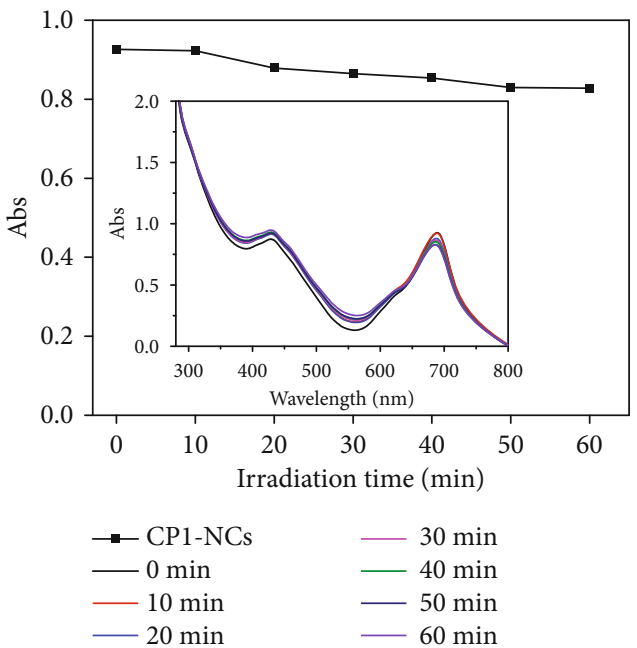

(d)

Figure 3: (a) TEM imaging of CP1-NCs. (b) DLS of CP1-NCs. (c) Absorption spectra of 6, CP1, and CP1-NCs. (d) Photostability of CP1-NCs. 
release process. The ${ }^{1} \mathrm{O}_{2}$ capture capability of CP1-NCs was investigated through the absorption decrease due to the EPO formation. Along with the increase of irradiation time, the absorption band at $254 \mathrm{~nm}$ decreased gradually, which demonstrated that ${ }^{1} \mathrm{O}_{2}$ sensitized by CP1-NCs was captured by itself to generate EPO (Figure S7). Under $690 \mathrm{~nm}$ irradiation, the CP1-NCs were able to sensitize oxygen to produce ${ }^{1} \mathrm{O}_{2}$, which displayed a decreasing in absorbance at $420 \mathrm{~nm}(\triangle \mathrm{Abs})$ in the presence of ${ }^{1} \mathrm{O}_{2}$ by 1,3diphenylisobenzofuran (DPBF) as an indicator (Figure S7(a)). Furthermore, the ${ }^{1} \mathrm{O}_{2}$ release of CP1-NCs was carried out under dark conditions at three temperatures of 37,45 , and $60^{\circ} \mathrm{C}$, respectively. As exhibited in Figure 2(f), a higher temperature could accelerate the release of ${ }^{1} \mathrm{O}_{2}$. In contrast, when 1,4-dimethylnaphthalene-free $\mathrm{CP} 3$-NPs were irradiated, the negligible $\Delta \mathrm{Abs}$ values were observed (Figure $\mathrm{S} 7(\mathrm{~b})$ ). The results suggested that $\mathrm{CP} 1-\mathrm{NCs}$ not only could effectively form EPO under irradiation but also release ${ }^{1} \mathrm{O}_{2}$ by the photothermal effect in aqueous solution. As shown in Figure 2(g), a hypothetical model further illustrated photothermal-induced release of CP1-NC under irradiation. Especially, compared to traditional photosensitizer, $\mathrm{O}_{2}$ is unnecessary in ${ }^{1} \mathrm{O}_{2}$ release process of CP1-NCs, suggesting great potential for enhancing the phototherapeutic performances of hypoxia-associative PDT. Therefore, the excellent PDT/PTT performances of CP1-NCs endowed it as potential phototherapy agents for the following NIR lightinduced therapy experiments.

2.4. Cytotoxicity Assay and PDT/PTT Efficiency of CP1-NCs. The cytotoxicity of CP1-NCs in vitro was explored through the 3-(4,5-dimethyl-2-thiazolyl)-2,5-diphenyl-2H-tetrazolium (MTT) assessment. HeLa cells were treated with CP1$\mathrm{NCs}$ at various concentrations $(1.0,2.0,4.0,6.0$, and $8.0 \mu \mathrm{g} \mathrm{mL}^{-1}$ ) for $24 \mathrm{~h}$ and then conducted with $690 \mathrm{~nm}$ light irradiation under $21 \%$ and $5 \% \mathrm{O}_{2}$ for $6 \mathrm{~min}$ or not, respectively (Figure 4(a)). The HeLa cell viability in the absence of irradiation was larger than $77 \%$ at the level of $8.0 \mu \mathrm{g} \mathrm{mL}^{-1}$, demonstrating the outstanding biocompatibility of CP1NCs. Furthermore, the cells cultured with ${ }^{1} \mathrm{O}_{2}$ loading $\mathrm{CP} 1$ NCs displayed relatively lower cell viability under $21 \%$ or $5 \% \mathrm{O}_{2}$ concentration, showing that the $\mathrm{CP} 1-\mathrm{NCs}$ could trigger cell oxidative damage and kill cells even under hypoxia condition. Owing to the intrinsic NIR emission of CP1NCs, the cellular uptake of CP1-NCs was further explored. The red fluorescence in the cytoplasm was found from the cells cultured with CP1-NCs through the confocal images, indicating the abundant uptake and excellent dispersion of CP1-NCs in HeLa cells (Figure S9). To investigate the phototherapy performance of the CP1-NCs including ${ }^{1} \mathrm{O}_{2}$ generation, $2^{\prime}, 7^{\prime}$-dichlorodihydrofluorescein diacetate (DCFH-DA) [65], which could be transformed into DCF with green luminescence in the presence of ${ }^{1} \mathrm{O}_{2}$, was used as $a^{1} \mathrm{O}_{2}$ indicator. As exhibited in Figure 4(b), the strong green luminescence was observed from the HeLa cells treated with CP1-NCs under $21 \%$ and $5 \% \mathrm{O}_{2}$ level, illustrating the production of abundant intracellular ${ }^{1} \mathrm{O}_{2}$ under irradiation. Moreover, the CP1-NCs generated negligible fluorescence in the absence of irradiation, since the physiological temperature (around $37^{\circ} \mathrm{C}$ ) was rather low so that it might not induce the release of ${ }^{1} \mathrm{O}_{2}$ from EPO. The results suggested that the release of ${ }^{1} \mathrm{O}_{2}$ from EPO might be triggered through the photothermal effect of CP1-NCs.

To explore the in vitro cell killing performance, calcein AM (live cells) and propidium iodide (dead cells) staining assessments were carried out under normoxia and hypoxia. When treated with CP1-NCs, the cells were dead under either $21 \%$ or $5 \%$ oxygen level with irradiation (Figure 4(c)). In the absence of light irradiation, the HeLa cells incubated with CP1-NCs mainly maintained alive under $21 \%$ and $5 \%$ oxygen levels. The results confirmed that the phototherapeutic performance was unaffected by the hypoxia. In addition, to demonstrate the cell population at different periods of apoptosis, flow cytometry assay was explored (Figures 4(d) and S10). Under $690 \mathrm{~nm}$ light irradiation, the significant improved cells were achieved in the fluorescein isothiocyanate- (FITC-) positive and PI-positive area under normoxia and hypoxia. The results demonstrated that the photothermal efficiency of CP1-NCs induced the release of ${ }^{1} \mathrm{O}_{2}$ and oxidative damage improved the phototherapeutic performances, motivating us to explore the application of CP1-NCs for enhanced tumor phototherapy.

2.5. In Vivo Luminescence Imaging, Photothermal Imaging, and DCFH-DA Staining of CP1-NCs. According to the NIR emission of CP1-NCs, the in vivo luminescence imaging of $\mathrm{CP} 1-\mathrm{NCs}$ was used to acquire the accurate therapeutic time. The CP1-NCs were intravenously injected into the tumorbearing mice, and their biodistributions were recorded at various postinjection time points. The luminescence signal in the tumor sites quickly increased from $2 \mathrm{~h}$ to $12 \mathrm{~h}$ after injection and decreased as the metabolism of CP1-NCs (Figure 5(a)). The signal intensity reached to its plateau at $12 \mathrm{~h}$ postinjection, which was acted as the reference time for the following therapy. In comparison, negligible or weak luminescence intensity was showed in other main organs (Figure S11). These imaging results suggested superior ability of the CP1-NCs to accumulate in tumor location, which was attributed to the EPR effect via the proper particle size.

To explore the performance of CP1-NCs to produce hyperthermia in vivo, CP1-NCs were injected into the mice at different doses, followed through infrared thermal imaging of mice after $12 \mathrm{~h}$ injection under $690 \mathrm{~nm}$ irradiation $\left(0.5 \mathrm{~W} \mathrm{~cm}^{-2}\right)$. PBS, as a control, led to a negligible temperature increase (Figure 5(b)). Under $690 \mathrm{~nm}$ irradiation, CP1NCs at the doses of $0.45,0.9$, and $1.8 \mathrm{mg} \mathrm{kg}^{-1} \mathrm{CP} 1$ triggered the tumor temperature changes of 11,19 , and $30^{\circ} \mathrm{C}$, respectively, suggesting remarkable temperature elevations with the increasing of dose (Figures 5(b)-5(d)). Because too high temperature increase produced detrimental influence for the normal cell, and too low temperature cannot obtain appropriate photothermal therapy efficiency, the level of CP1-NCs at $0.9 \mathrm{mg} \mathrm{kg}^{-1} \mathrm{CP} 1$ was selected for the subsequent tumor therapy. Furthermore, the capability of CP1-NCs producing ROS at the tumor slice was investigated by the DCFH-DA staining under $690 \mathrm{~nm}$ light irradiation. Vitamin $\mathrm{C}(\mathrm{VC})$ as a ${ }^{1} \mathrm{O}_{2}$ scavenger was used to scavenge ${ }^{1} \mathrm{O}_{2}$. As 

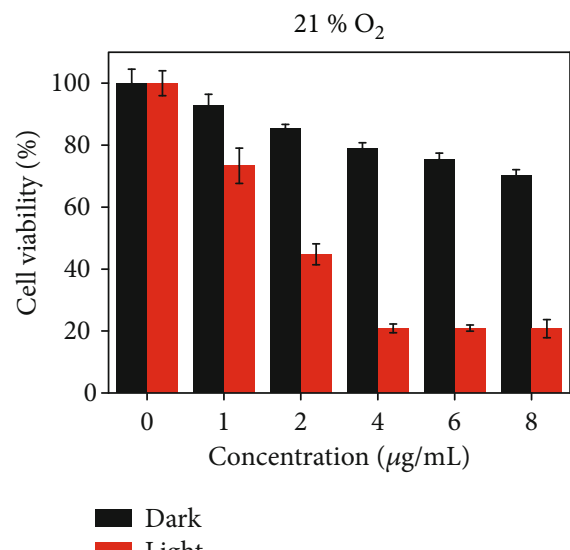

(a)
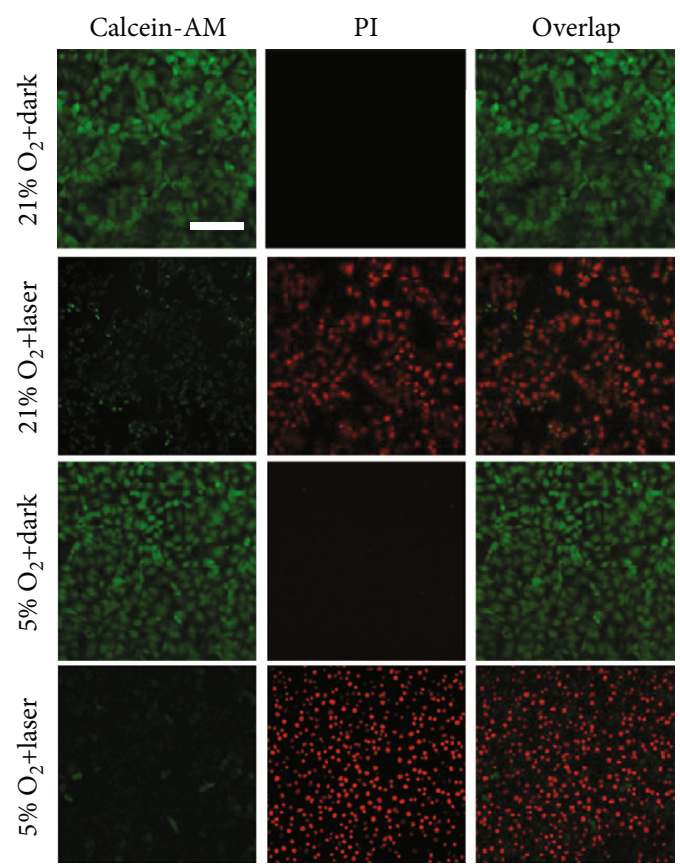

(c)
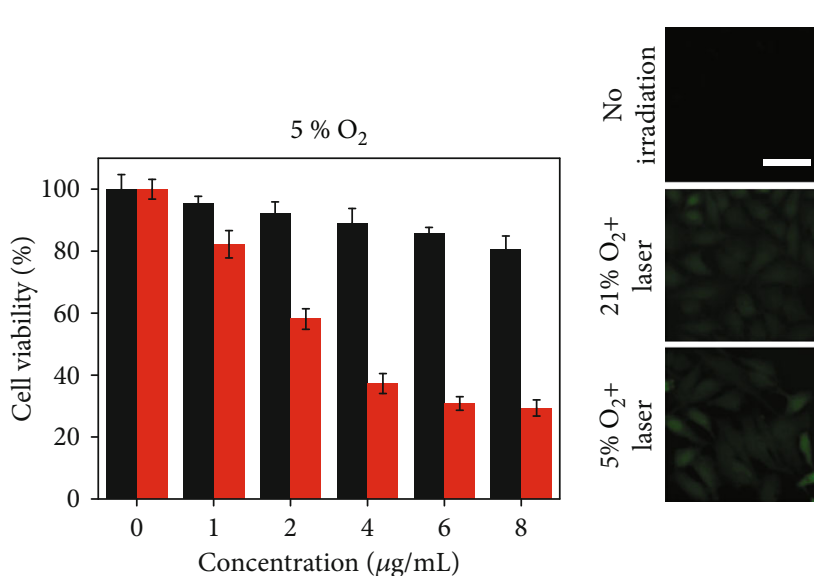

Bright field

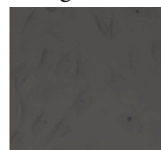

Overlap

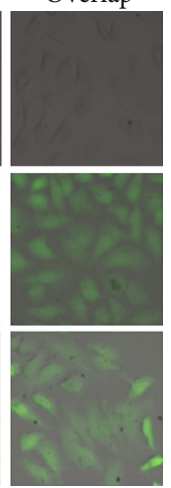

(b)

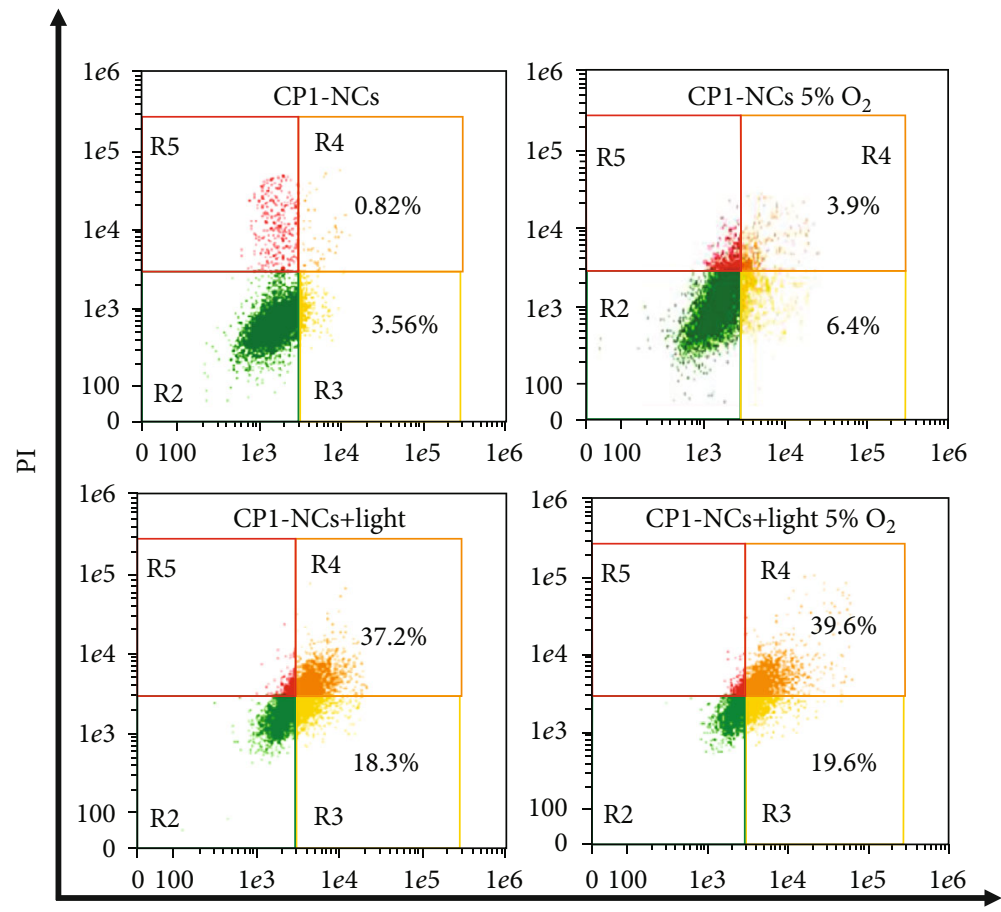

FITC

(d)

FIGURE 4: In vitro evaluation of CP1-NCs under $21 \%$ or $5 \%$ oxygen concentration. (a) MTT assay of CP1-NCs with and without irradiation. (b) Confocal image of HeLa cells cultured with CP1-NCs with or without 5 min light irradiation $\left(690 \mathrm{~nm}, 0.5 \mathrm{~W} \mathrm{~cm}{ }^{-2}\right)$ using DCFH-DA staining $\left(\lambda_{\mathrm{ex}}=488 \mathrm{~nm}, \lambda_{\mathrm{em}}=500-540 \mathrm{~nm}\right.$, scale bar: $\left.100 \mu \mathrm{m}\right)$. (c) Calcein AM and PI-stained HeLa tumor cells treated with CP1-NCs with or without $5 \mathrm{~min}$ light irradiation $\left(690 \mathrm{~nm}, 0.5 \mathrm{~W} \mathrm{~cm}^{-2}\right.$, scale bar: $\left.100 \mu \mathrm{m}\right)$. (d) Flow cytometry quantification of apoptosis of HeLa cells incubated with CP1-NCs under $690 \mathrm{~nm}$ light irradiation $\left(0.5 \mathrm{~W} \mathrm{~cm}^{-2}\right)$.

displayed in Figure 5(e), the strong green luminescence was found at the tumor slice of mice cultured with CP1NCs under light irradiation. However, the HeLa cells with CP1-NCs without light irradiation or in the presence of $\mathrm{VC}$ induced negligible green luminescence. The results suggested that $\mathrm{CP} 1-\mathrm{NCs}$ could generate abundant ${ }^{1} \mathrm{O}_{2}$ under irradiation.
2.6. Synergistic Phototherapy. Encouraged by the preferable luminescence imaging, sustainable ${ }^{1} \mathrm{O}_{2}$ production, and good photothermal conversion efficiency of CP1-NCs, the phototherapy effect of CP1-NCs was carried out in vivo using the HeLa tumor mouse model. After the tumor volume increased around $120 \mathrm{~mm}^{3}$, the mice were separated into four groups. The mice were treated with (I) PBS, (II) CP1-NCs only, 
$0 \mathrm{~h}$
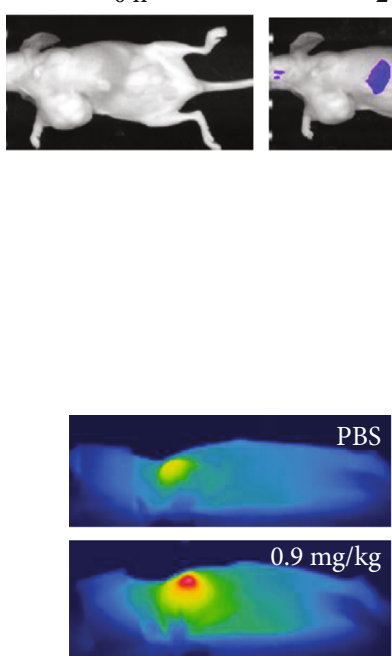

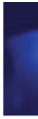
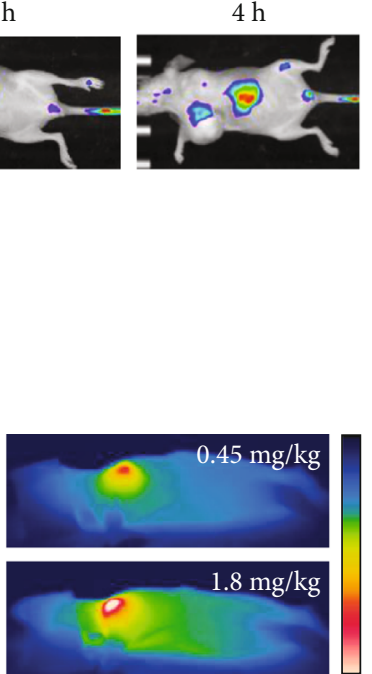

$12 \mathrm{~h}$
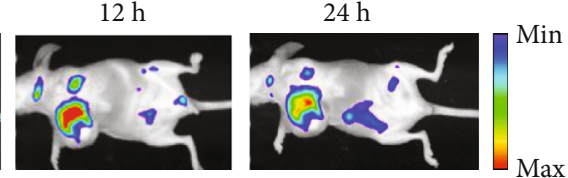

(a)

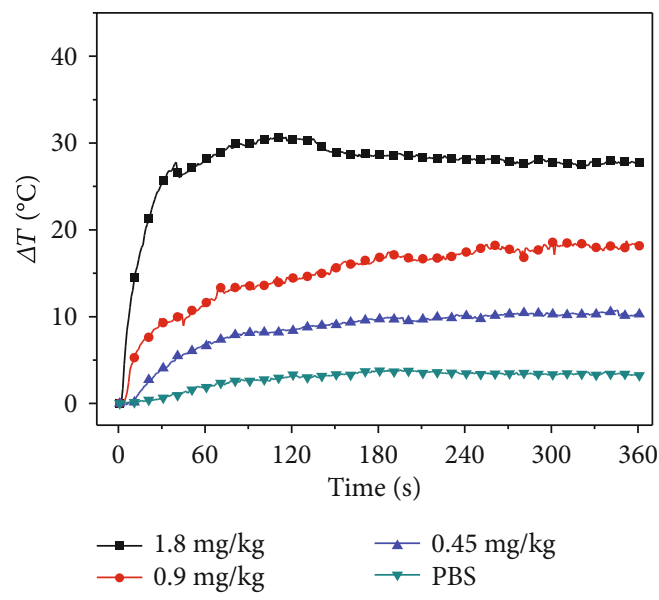

(c)$$
\begin{aligned}
& \longrightarrow 0.45 \mathrm{mg} / \mathrm{kg} \\
& \rightarrow \text { PBS }
\end{aligned}
$$

(b)

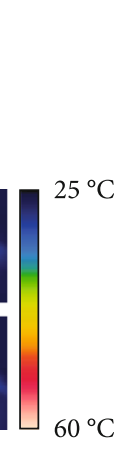

Time (min)

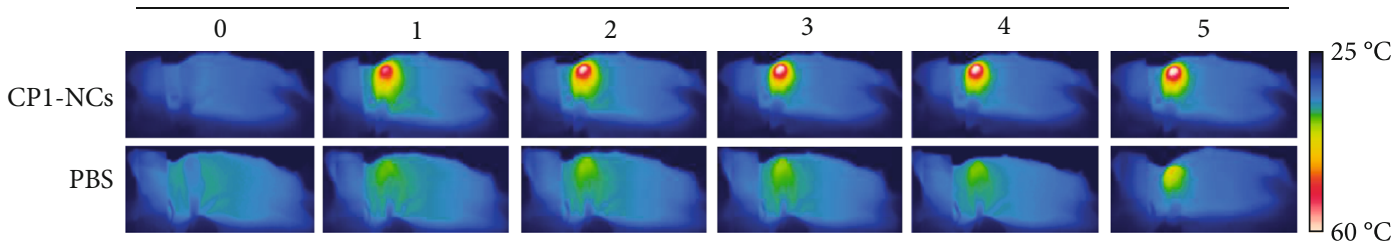

(d)
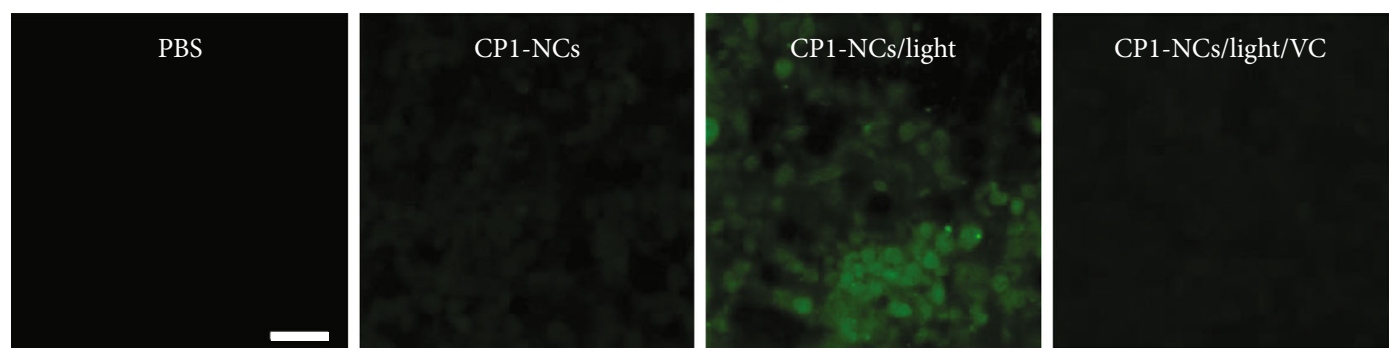

(e)

FIGURE 5: In vivo photothermal performances and production of singlet oxygen of CP1-NCs. (a) Luminescence image of the HeLa tumorbearing mice at various time points through tail intravenous injection of CP1-NCs ( $n=3$ mice per group). (b) Infrared thermograph of the mice bearing HeLa tumor treated with CP1-NCs at different concentrations under $690 \mathrm{~nm}$ irradiation at $0.5 \mathrm{~W} \mathrm{~cm}$ for $6 \mathrm{~min}$ and (c) the corresponding temperature elevation within tumor region. (d) Representative thermal images of mice (tumor sites) subjected to $690 \mathrm{~nm}$ light irradiation for $12 \mathrm{~h}$ postinjection of CP1-NCs $\left(0.9 \mathrm{mg} \mathrm{kg}^{-1}, 120 \mu \mathrm{L}\right)$ and PBS. (e) DCFH-DA staining of tumor slice from the mice treated with CP1-NCs at $12 \mathrm{~h}$ postinjection in the absence or presence of VC under light irradiation or not (scale bar: $100 \mu \mathrm{m})$.

(III) $\mathrm{CP} 1-\mathrm{NCs} \quad\left(150 \mu \mathrm{L}, \quad 0.9 \mathrm{mg} \mathrm{kg}^{-1} \quad \mathrm{CP} 1\right)+$ irradiation $\left(690 \mathrm{~nm}, 0.5 \mathrm{~W} \mathrm{~cm}^{-2}\right)+\mathrm{VC}$, and (IV) CP1-NCs $(150 \mu \mathrm{L}$, $\left.0.9 \mathrm{mg} \mathrm{kg}^{-1} \mathrm{CP} 1\right)+$ irradiation $\left(690 \mathrm{~nm}, 0.5 \mathrm{~W} \mathrm{~cm}^{-2}\right)$, respectively. The fourth group acted as the therapy group. The IIII groups served as control. The mouse tumor volume and weight were recorded every two days. For groups (III) and (IV), 6 min irradiation was implemented after $12 \mathrm{~h}$ injection. As exhibited in Figure 6(a), the tumor volumes of (I) and (II) exhibited an expeditious growth in the absence of irradiation, demonstrating the excellent biocompatibility of CP1-NCs.
Compared with tumors treated with CP1-NCs+irradiation $+\mathrm{VC}$, the tumor was significantly inhibited for those treated with CP1-NCs+irradiation, suggesting that the continuous delivery of ${ }^{1} \mathrm{O}_{2}$ leads to better phototherapy performance. Similar body weight change was observed in the control groups in comparison with the treatment group, suggesting the negligible side effects of CP1-NCs (Figure 6(b)). The photo of tumors was consistent with the monitored tumor weight (Figures 6(c) and 6(d)). The therapy results confirmed that CP1-NCs can achieve enhanced PDT performance 


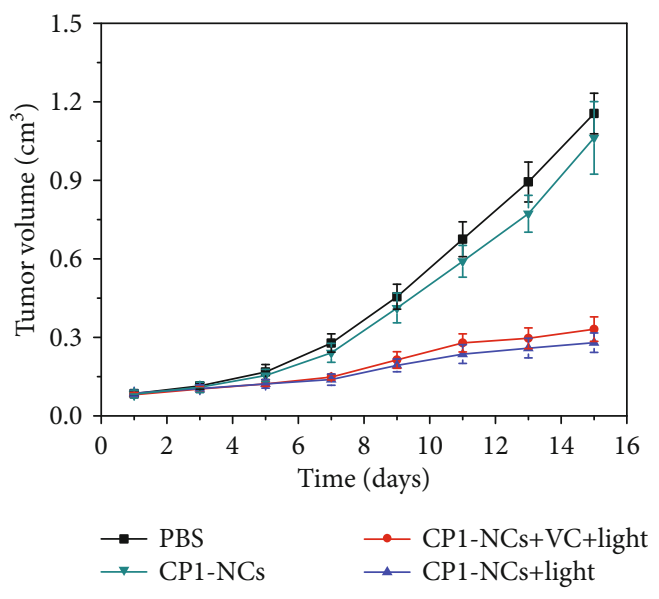

(a)

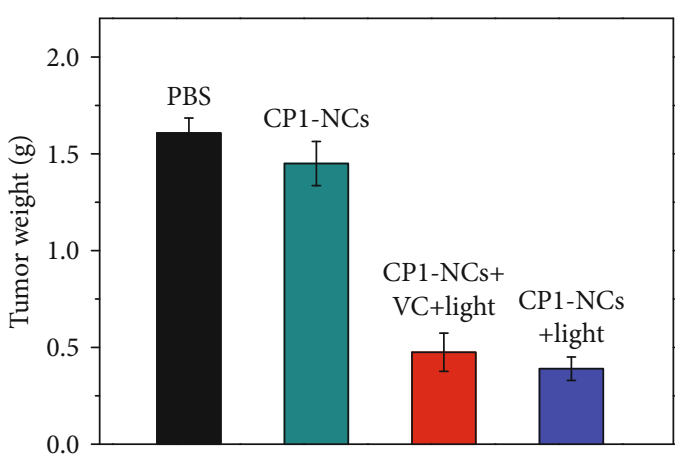

(c)

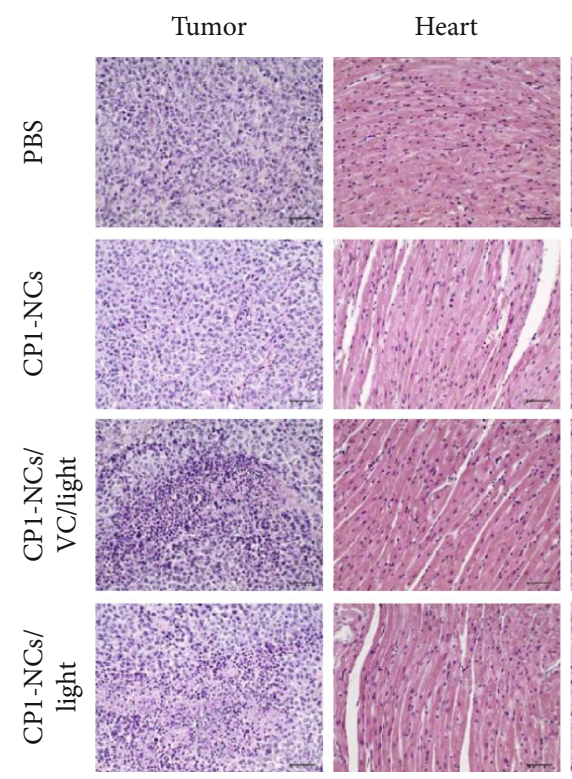

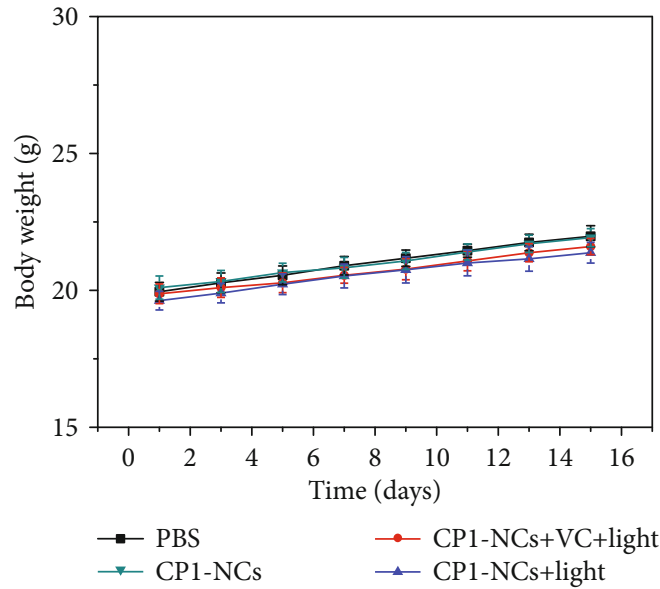

(b)

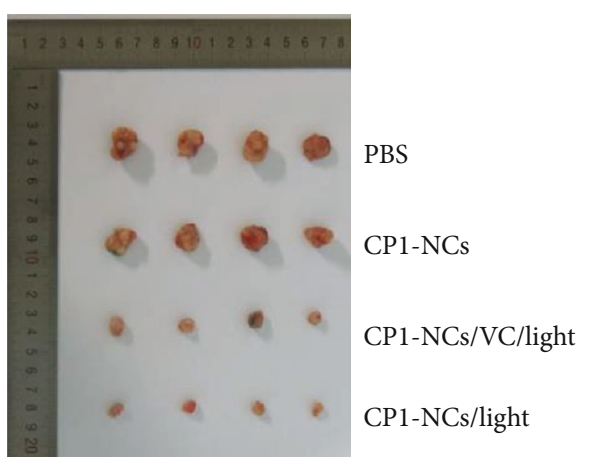

(d)
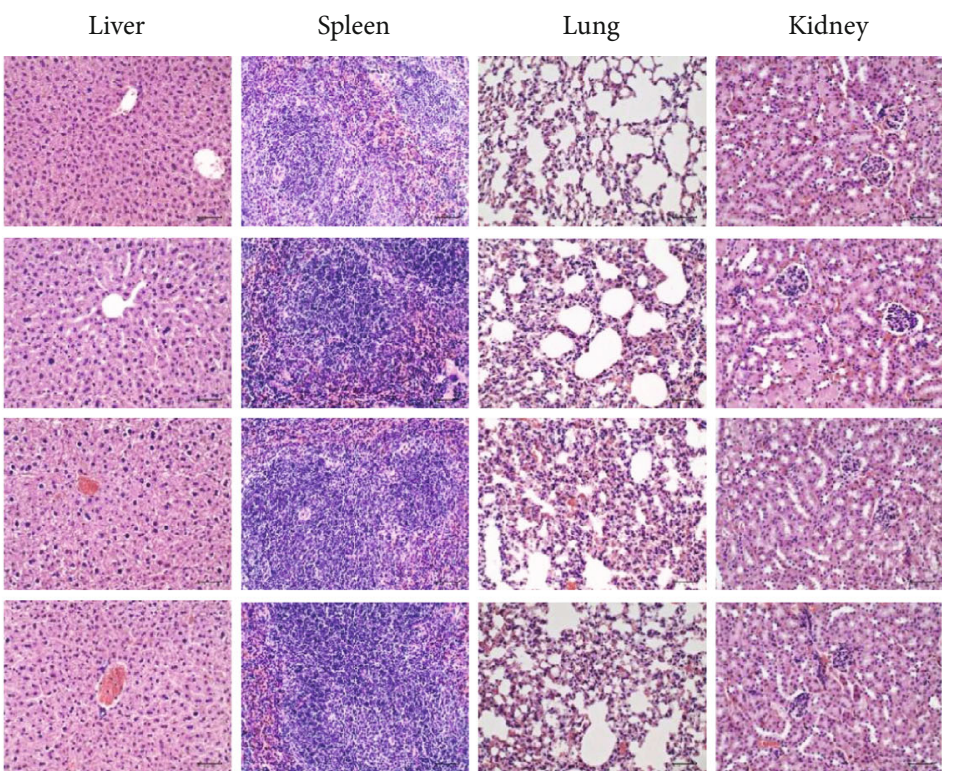

(e)

FIGURE 6: (a) Relative tumor volume changes of mice with various therapies. (b) Body weight changes of mice with different therapies. (c) Tumor weight of mice with different treatments. (d) Photos of each group of mice after the treatment. (e) H\&E staining of the tumor, heart, liver, spleen, lung, and kidney obtained from the tumor-bearing mice with different treatment groups (scale bar: $100 \mu \mathrm{m}$ ).

through the ${ }^{1} \mathrm{O}_{2}$ release for inhibiting tumor growth, which could act as a potential phototherapy agent for precision tumor ablation. Furthermore, to explore the dark toxicity of
CP1-NCs, the hematoxylin and eosin (H\&E) analysis of the tumor and major organs (heart, liver, spleen, lung, and kidney) acquired from the mice was measured after therapy. 

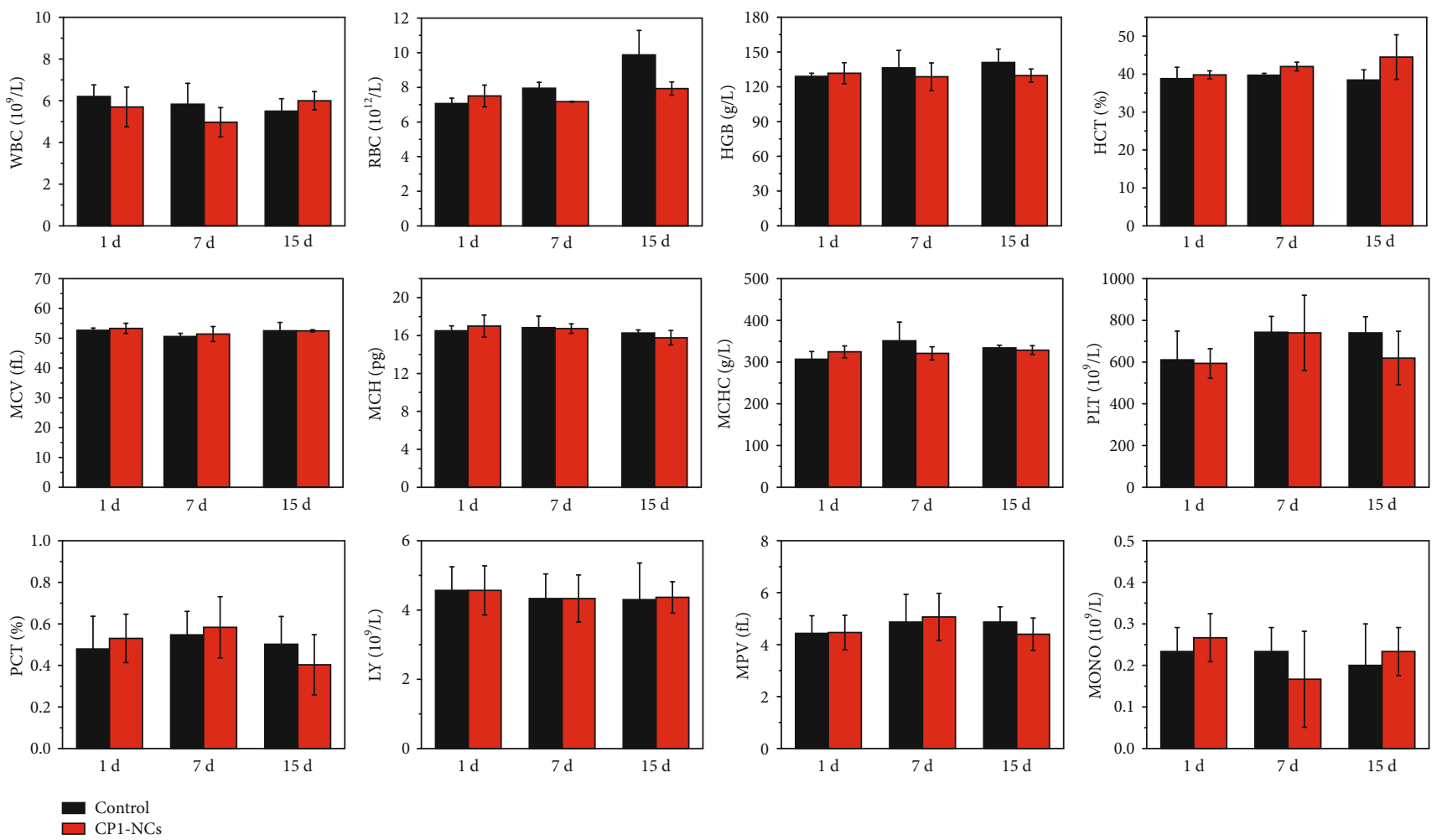

FIGURE 7: Blood hematology analysis from healthy and CP1-NC-treated mice performed at 1, 7, and 15 days. The hematology indicators include white blood cell (WBC), red blood cell (RBC), hemoglobin (HGB), hematocrit (HCT), mean corpuscular volume (MCV), mean corpuscular hemoglobin $(\mathrm{MCH})$, mean corpuscular hemoglobin concentration (MCHC), platelet (PLT), plateletcrit (PCT), lymphocyte (LY), mean platelet volume (MPV), and monocyte count (MONO). Error bars correspond to standard deviation for $n=3$.

The pathomorphology analysis indicated similar morphological properties with negligible cell and tissue damage in the major organs for all mice (Figure 6(e)). The results validated that CP1-NCs could not generate significant photodamage for normal organs, although they might accumulated there.

To explore the biological toxicity of CP1-NCs, blood routine analysis and blood biochemistry were carried out. No significant changes of the blood biochemistry (Figure S12) and blood hematology (Figure 7) during the phototherapy process were found, suggesting that the CP1-NCs possess excellent biocompatibility.

\section{Conclusion}

In summary, we reported a new photothermally responsive conjugated polymeric ${ }^{1} \mathrm{O}_{2}$ carrier for phase changecontrolled and luminescence imaging-guided sustainable phototherapy for hypoxic tumor. The CP1-NCs not only can serve as NIR luminescence imaging agent for real-time identification of the nanocarrier accumulation but also can trap the ${ }^{1} \mathrm{O}_{2}$ produced from CP1 under irradiation and form a stable EPO. The EPO undergoes cycloreversion to release ${ }^{1} \mathrm{O}_{2}$ through the NIR-triggered photothermal efficiency of CP1 and controlled phase change of PCM, which can be used for oxygen-independent PDT for relieving tumor hypoxic microenvironment. As compared with CP1-NCs plus VC, the CP1-NCs exhibit better efficiency in inhibiting tumor growth through $1 \mathrm{O} 2$ release for enhanced cancer photother- apy. Therefore, the present study demonstrates the potential of CP1-NCs in controlled and sustainable cancer phototherapy under hypoxia, affording a new strategy for developing oxygen-independent cancer phototherapy platform.

\section{Materials and Methods}

4.1. Materials. Unless otherwise stated, all raw materials were bought and utilized as received. Oleic acid and 1-hexadecanol were obtained from Aldrich chemistry. The biocompatible $\alpha$ lecithin and amphiphilic DSPE-mPEG 5000 were purchased from Adamas-Beta. Calcein AM/PI stain kit was obtained from Nanjing KeyGen Biotech Co., Ltd. The detailed synthesis of intermediates and CPs can be found in the Supporting Information. Oleic acid and 1-hexadecanol were weighed at the various mass ratios (OA : Hex $=1: 2.5,3.0,3.5,4.0$, and 4.5) and then dissolved in ethanol. Then, the mixture solution was ultrasonically mixed and stored in a refrigerator as PCM with controllable melting point.

4.2. Preparation and Characterization of CP-NPs. The CPNPs were obtained through a resolidification method. The CPs and PCM $(2 \mathrm{mg} / \mathrm{mL})$ dissolved in THF solution serve as solution 1. The DSPE-mPEG 5000 and $\alpha$-lecithin $(10 \mathrm{mg}$ in $10 \mathrm{~mL}$ water) dispersed in aqueous solution were used as solution 2 . Then, the two solutions were mixed under continuous sonication at $50^{\circ} \mathrm{C}$ for $6 \mathrm{~min}$ and then rapidly cooled in an ice bath. After the solution was warmed up to room 
temperature, THF was removed under stirring. The solution was filtered via a polyethersulfone (PES) syringe-driven filter $(0.44 \mu \mathrm{m})$ and was further centrifuged utilizing a centrifugal filter. The concentration of CP-NPs was determined according to absorption spectra. The morphology and particle size of CP-NPs were measured by transmission electron microscopy and dynamic light scattering.

4.3. Singlet Oxygen Test. To explore the singlet oxygen production and photodynamic improvement of CP-NPs, DPBF served as an ${ }^{1} \mathrm{O}_{2}$ indicator. CP-NPs at different concentrations were mixed with DPBF in water solution under continuous stirring. Under $690 \mathrm{~nm}$ irradiation, the absorption spectra of DPBF were acquired.

4.4. Photothermal Performance. The temperature elevation of CP-NCs at different concentrations $\left(75,150\right.$, and $300 \mu \mathrm{g} \mathrm{mL}^{-}$ ${ }^{1}$ ) under $690 \mathrm{~nm}$ irradiation $\left(0.5 \mathrm{~W} \mathrm{~cm}^{-2}, 6 \mathrm{~min}\right)$ was recorded by the FLIR E40. To acquire the photothermal conversion efficiency, CP1-NCs were exposed under $690 \mathrm{~nm}$ light irradiation at $0.5 \mathrm{~W} \mathrm{~cm}^{-2}$. After the temperature reached a plateau, the light irradiation was removed for cooling down to ambient temperature.

4.5. Cellular Experiment. The cells were treated in Dulbecco's modified Eagle medium and provided with $10 \%$ fetal bovine serum (FBS) at $37^{\circ} \mathrm{C}$ with $5 \% \mathrm{CO}_{2}$. To evaluate the cellular uptake, HeLa cells were incubated with CP1-NCs in the dark for $6 \mathrm{~h}$. Then, the cells were washed with fresh PBS twice before being imaged by confocal imaging. To test the phototoxicity of CP1-NCs, HeLa cells were treated with PBS or CP1-NCs under $21 \%$ or $5 \%$ oxygen concentration for $24 \mathrm{~h}$, followed by $5 \mathrm{~min}$ light irradiation under $690 \mathrm{~nm}$ $\left(0.5 \mathrm{~W} \mathrm{~cm}^{-2}\right)$. The HeLa cells were incubated with DCFH$\mathrm{DA}$ at $37^{\circ} \mathrm{C}$ under $21 \%$ or $5 \%$ oxygen level for $30 \mathrm{~min}$, followed by $690 \mathrm{~nm}$ irradiation at $0.5 \mathrm{~W} \mathrm{~cm}^{-2}$ for $5 \mathrm{~min}$. Furthermore, to explore the cell population at different stages of apoptosis, AM/PI and flow cytometry assay was carried out under normoxia and hypoxia.

4.6. In Vivo Fluorescence Imaging. For in vivo luminescence imaging, HeLa tumor-bearing mice were intravenously injected with CP1-NCs. The luminescence signals of the mice were collected $\left(\lambda_{\text {ex }}=690 \mathrm{~nm}\right)$ through an IVIS Lumina $K$ in vivo imaging system (PerkinElmer) using a xenon lamp which was equipped with different long- and band-pass filters. An Andor EMCCD-DU897 camera was used as an imaging detector. Images were taken at 2, 4, 8, 12, and $24 \mathrm{~h}$ postinjection. After $24 \mathrm{~h}$, the tumor and major organs were collected and the fluorescence intensity was analyzed to confirm the accurate therapeutic time.

4.7. Blood Routine Examination. The whole blood was acquired from the orbital venous plexus on 1-, 7-, and 15day postinjection and subject to blood routine test. The blood samples were acquired from the mouse fundus artery in each group. Blood solutions $(100 \mu \mathrm{L})$ were treated with anticoagulant for hematology analysis. After being kept at $4^{\circ} \mathrm{C}$ for $4 \mathrm{~h}$ and centrifuged, blood plasma samples $(200 \mu \mathrm{L})$ were obtained from blood for biochemistry assay.
4.8. In Vivo Synergistic Therapy. All tumor nude mice were acquired from Jiangsu KeyGen Biotech Co., Ltd. and used referring to the standard of the Laboratory Animal Center of Jiangsu KeyGen Biotech Co., Ltd. When the tumor volume increased to around $120 \mathrm{~mm}^{3}$, we began to carry out the phototherapy experiments. To assess the phototherapy effect of CP1-NCs, the tumor-bearing HeLa mice were injected with CP1-NCs at a dose of $0.9 \mathrm{mg} \mathrm{kg}^{-1}$. The tumors were exposed under $690 \mathrm{~nm}$ light irradiation at $0.5 \mathrm{~W} \mathrm{~cm}^{-2}$ for $6 \mathrm{~min}$.

4.9. Histological Staining. After treatment, the final tumor and major organs were fixed with $4 \%$ formaldehyde for $\mathrm{H} \& \mathrm{E}$ staining to explore the side effect of CP1-NCs.

\section{Conflicts of Interest}

The authors declare no competing financial interests.

\section{Authors' Contributions}

Guo Li, Qiang Zhao, Shujuan Liu, and Wei Huang designed the project. Guo Li carried out the material synthesis and characterizations. Guo Li, Shujuan Liu, and Qiang Zhao analyzed the whole project as well as wrote the manuscript. Ruyi Zhou and Bo Yu performed the in vitro experiments. Weili Zhao and Jie Zhou carried out the cell measurement. All the authors were included in the discussion and approved the manuscript.

\section{Acknowledgments}

This work was supported by the China National Funds for Distinguished Young Scientists (61825503), the National Natural Science Foundation of China (61775101 and 61805122), the National Key Research and Development Program of China (2017YFA0205302), and the Open Research Fund of State Key Laboratory of Bioelectronics, Southeast University.

\section{Supplementary Materials}

Figure S1: synthetic routes of the 1-7. Figure S2: synthetic routes of the CP1-CP3. Figure S3: morphological changes of the CP-NCs before and after NIR irradiation. The size of the CP1-NCs@PCM became smaller after the irradiation, while no obvious changes were obtained from the CP2NPs@PCM. Scale bars: 200 nm. Figure S4: (a) UV-vis-NIR absorption spectra of $\mathrm{CP} 1$ at various concentrations in $\mathrm{CH}_{2} \mathrm{Cl}_{2}$. (b) The equation was calculated according to the maximal absorption of (a). (c) UV-vis-NIR absorption spectra of $\mathrm{CP} 2$ at various concentrations in $\mathrm{CH}_{2} \mathrm{Cl}_{2}$. (d) The equation was calculated according to the maximal absorption of (c). (e) UV-vis-NIR absorption spectra of CP3 at various concentrations in $\mathrm{CH}_{2} \mathrm{Cl}_{2}$. (f) The equation was calculated according to the maximal absorption of (e). Figure S5: absorption spectra of 7, CPs, and CP-NPs. Figure S6: emission spectra of $\mathrm{CP} 1-\mathrm{CP} 3$ in $\mathrm{CH}_{2} \mathrm{Cl}_{2}$ and $\mathrm{CP}-\mathrm{NPs}$ in water. Figure S7: (a) absorption changes of DPBF with CP1-NCs under $690 \mathrm{~nm}$ irradiation in water and (inset) the absorption decrease owing to the endoperoxide formation. (b) $\triangle \mathrm{Abs}$ of $\mathrm{DPBF}$ in mixture solution of CP3-NPs and DBPF. Figure 
S8: (a) photothermal stability of CP1-NCs. (b) The heating curve of the CP1-NCs in a procedure of laser-on and laseroff. (c) The linear cooling time data versus $-\ln (\theta)$ acquired from the cooling period of (b). (d) Temperature elevation of CP2-NPs. Figure S9: confocal images of the cellular uptake of CP1-NCs and DAPI. Scale bar: $20 \mathrm{~nm}$. Figure S10: flow cytometry quantification of annexin V-FITC and PI-labeled HeLa cells cultured with only PBS or laser, respectively. Figure S11: the CP1-NC distribution in the tumor and major organs after $12 \mathrm{~h}$ intravenous injection. Figure S12: blood biochemical assay. (a) Glutamic-pyruvic transaminase (ALT), (b) glutamic oxalacetic transaminase (AST), (c) urea nitrogen (BUN), and (d) creatinine (CREA) concentrations for liver and kidney functions of healthy nude mice 15 days after tail intravenous injection of CP1-NCs. Error bars represent the standard deviations $(n=3)$. Figure S13: the ${ }^{1} \mathrm{H}$ NMR spectrum of 6 in $\mathrm{CDCl}_{3}$. Figure S14: the ${ }^{13} \mathrm{C}$ NMR spectrum of 6 in $\mathrm{CDCl}_{3}$. Figure S15: the ${ }^{1} \mathrm{H}$ NMR spectrum of 7 in $\mathrm{CDCl}_{3}$. Figure S16: the ${ }^{1} \mathrm{H}$ NMR spectra of CP1-CP3. Figure S17: MALDI-TOF-MS spectra of 4-6. (Supplementary Materials)

\section{References}

[1] W. Fan, P. Huang, and X. Chen, "Overcoming the Achilles' heel of photodynamic therapy," Chemical Society Reviews, vol. 45, no. 23, pp. 6488-6519, 2016.

[2] Z. Zhou, J. Song, L. Nie, and X. Chen, "Reactive oxygen species generating systems meeting challenges of photodynamic cancer therapy," Chemical Society Reviews, vol. 45, no. 23, pp. 6597-6626, 2016.

[3] Y. Wan, G. Lu, J. Zhang et al., "A biocompatible free radical nanogenerator with real-time monitoring capability for high performance sequential hypoxic tumor therapy," Advanced Functional Materials, vol. 29, no. 39, article 1903436, 2019.

[4] W. Lv, Z. Zhang, K. Y. Zhang et al., "A mitochondria-targeted photosensitizer showing improved photodynamic therapy effects under hypoxia," Angewandte Chemie International Edition, vol. 55, no. 34, pp. 9947-9951, 2016.

[5] L. Jiang, H. Bai, L. Liu, F. Lv, X. Ren, and S. Wang, "Hemoglobin-linked conjugated polymer nanoparticles for selfluminescing and oxygen self-supplying phototherapy," Angewandte Chemie International Edition, vol. 58, no. 32, pp. 10770-10775, 2019.

[6] J. Kim, H. R. Cho, H. Jeon et al., "Continuous $\mathrm{O}_{2}$-evolving $\mathrm{MnFe}_{2} \mathrm{O}_{4}$ Nanoparticle-Anchored mesoporous silica nanoparticles for efficient photodynamic therapy in hypoxic cancer," Journal of the American Chemical Society, vol. 139, no. 32, pp. 10992-10995, 2017.

[7] M. Li, S. Long, Y. Kang et al., "De novo design of phototheranostic sensitizers based on structure-inherent targeting for enhanced cancer ablation," Journal of the American Chemical Society, vol. 140, no. 46, pp. 15820-15826, 2018.

[8] V.-N. Nguyen, S. Qi, S. Kim et al., "An emerging molecular design approach to heavy-atom-free photosensitizers for enhanced photodynamic therapy under hypoxia," Journal of the American Chemical Society, vol. 141, no. 41, pp. 1624316248, 2019.

[9] Z. Wang, Y. Zhang, E. Ju et al., "Biomimetic nanoflowers by self-assembly of nanozymes to induce intracellular oxidative damage against hypoxic tumors," Nature Communications, vol. 9, no. 3, article 3334, 2018.

[10] J. M. Brown and W. R. Wilson, "Exploiting tumour hypoxia in cancer treatment," Nature Review Cancer, vol. 4, no. 6, pp. 437-447, 2004.

[11] J. Shen, J. Chen, Z. Ke, D. Zou, L. Sun, and J. Zou, "Heavy atom-free semiconducting polymer with high singlet oxygen quantum yield for prostate cancer synergistic phototherapy," Materials Chemistry Frontiers, vol. 3, no. 6, pp. 1123-1127, 2019.

[12] Y. C. Tsai, P. Vijayaraghavan, W. H. Chiang et al., "Targeted delivery of functionalized upconversion nanoparticles for externally triggered photothermal/photodynamic therapies of brain glioblastoma," Theranostics, vol. 8, no. 5, pp. 1435$1448,2018$.

[13] J. Zou, Z. Yin, P. Wang et al., "Photosensitizer synergistic effects: D-A-D structured organic molecule with enhanced fluorescence and singlet oxygen quantum yield for photodynamic therapy," Chemical Science, vol. 9, no. 8, pp. 21882194, 2018.

[14] R. Li, C. Zhang, B. Xie et al., "A two-photon excited $\mathrm{O}_{2}$-evolving nanocomposite for efficient photodynamic therapy against hypoxic tumor," Biomaterials, vol. 194, pp. 84-93, 2019.

[15] Y. Dai, C. Xu, X. Sun, and X. Chen, "Nanoparticle design strategies for enhanced anticancer therapy by exploiting the tumour microenvironment," Chemical Society Reviews, vol. 46, no. 12, pp. 3830-3852, 2017.

[16] D. Wang, H. Wu, W. Q. Lim et al., "A mesoporous nanoenzyme derived from meta-organic frameworks with endogenous oxygen generation to alleviate tumor hypoxia for significantly enhanced photodynamic therapy," Advanced Materials, vol. 31, no. 27, article 1901893, 2019.

[17] M. Huo, L. Wang, L. Zhang, C. Wei, Y. Chen, and J. Shi, "Photosynthetic tumor oxygenation by photosensitized cyanobacterial cells for enhanced photodynamic therapy," Angewandte Chemie International Edition, vol. 59, no. 5, pp. 1909-1913, 2020.

[18] J.-N. Liu, W. Bu, and J. Shi, "Chemical design and synthesis of functionalized probes for imaging and treating tumor hypoxia," Chemical Society Reviews, vol. 117, no. 9, pp. 6160-6224, 2017.

[19] Z. Wang, Y. Ju, Z. Ali et al., "Near-infrared light and tumor microenvironment dual responsive size-switchable nanocapsules for multimodal tumor theranostics," Nature Communications, vol. 10, no. 2, article 4418, 2019.

[20] M. Li, J. Xia, R. Tian et al., "Near-infrared light-initiated molecular superoxide radical generator: rejuvenating photodynamic therapy against hypoxic tumors," Journal of the American Chemical Society, vol. 140, no. 44, pp. 14851-14859, 2018.

[21] G. Yang, S. Z. F. Phua, W. Q. Lim et al., "A hypoxia-responsive albumin-based nanosystem for deep tumor penetration and excellent therapeutic efficacy," Advanced Matericals, vol. 31, no. 25, p. 1901513, 2019.

[22] Y. Bao, X. Hua, J. Zeng, and F. Wu, "Bacterial template synthesis of multifunctional nanospindles for glutathione detection and enhanced cancer-specific chemo-chemodynamic therapy," Research, vol. 2020, article 9301215, pp. 1-15, 2020.

[23] W. Xiu, S. Gan, Q. Wen et al., "Biofilm microenvironmentresponsive nanotheranostics for dual-mode imaging and hypoxia-relief-enhanced photodynamic therapy of bacterial infections," Research, vol. 2020, article 9426453, pp. 1-15, 2020. 
[24] D. Cui, J. Huang, X. Zhen, J. Li, Y. Jiang, and K. Pu, “A semiconducting polymer nano-prodrug for hypoxia-activated photodynamic cancer therapy," Angewandte Chemie International Edition, vol. 58, no. 18, pp. 5920-5924, 2019.

[25] C. Ji, Q. Gao, X. Dong et al., "A size-reducible nanodrug with an aggregation-enhanced photodynamic effect for deep chemo-photodynamic therapy," Angewandte Chemie International Edition, vol. 57, no. 35, pp. 11384-11388, 2018.

[26] J. Li, D. Cui, Y. Jiang, J. Huang, P. Cheng, and K. Pu, "Nearinfrared photoactivatable semiconducting polymer nanoblockaders for metastasis-inhibited combination cancer therapy," Advanced Matericals, vol. 31, no. 46, article 1905091, 2019.

[27] Y. Cheng, H. Cheng, C. Jiang et al., "Perfluorocarbon nanoparticles enhance reactive oxygen levels and tumour growth inhibition in photodynamic therapy," Nature Communications, vol. 6, no. 1, article 8785, 2015.

[28] Q. Yu, T. Huang, C. Liu et al., "Oxygen self-sufficient NIRactivatable liposomes for tumor hypoxia regulation and photodynamic therapy," Chemical Science, vol. 10, no. 39, pp. 90919098, 2019.

[29] H. Zhu, J. Li, X. Qi, P. Chen, and K. Pu, "Oxygenic hybrid semiconducting nanoparticles for enhanced photodynamic therapy," Nano Letters, vol. 18, no. 1, pp. 586-594, 2018.

[30] H. Chen, J. Tian, W. He, and Z. Guo, " $\mathrm{H}_{2} \mathrm{O}_{2}$-activatable and $\mathrm{O}_{2}$-evolving nanoparticles for highly efficient and selective photodynamic therapy against hypoxic tumor cells," Journal of the American Chemical Society, vol. 137, no. 4, pp. 15391547, 2015.

[31] B. Ma, S. Wang, F. Liu et al., "Self-assembled copper-amino acid nanoparticles for in situ glutathione and $\mathrm{H}_{2} \mathrm{O}_{2}$ sequentially triggered chemodynamic therapy," Journal of the American Chemical Society, vol. 141, no. 2, pp. 849-857, 2019.

[32] L.-S. Lin, T. Huang, J. Song et al., "Synthesis of copper peroxide nanodots for $\mathrm{H}_{2} \mathrm{O}_{2}$ self-supplying chemodynamic therapy," Journal of the American Chemical Society, vol. 141, no. 25, pp. 9937-9945, 2019.

[33] Z. Tang, H. Zhang, Y. Liu et al., "Antiferromagnetic pyrite as the tumor microenvironment-mediated nanoplatform for self-enhanced tumor imaging and therapy," Advanced Materials, vol. 29, no. 47, article 1701683, 2017.

[34] H. Wang, B. Lv, Z. Tang et al., "Scintillator-based nanohybrids with sacrificial electron prodrug for enhanced X-ray-induced photodynamic therapy," Nano Letters, vol. 18, no. 9, pp. 5768-5774, 2018.

[35] Y. Liu, W. Zhen, L. Jin et al., "All-in-one theranostic nanoagent with enhanced reactive oxygen species generation and modulating tumor microenvironment ability for effective tumor eradication," ACS Nano, vol. 12, no. 5, pp. 48864893, 2018.

[36] Y. Liu, Y. Jiang, M. Zhang, Z. Tang, M. He, and W. Bu, "Modulating hypoxia via nanomaterials chemistry for efficient treatment of solid tumors," Accounts of Chemical Research, vol. 51, no. 10, pp. 2502-2511, 2018.

[37] T. Yang, L. Liu, Y. Deng et al., "Ultrastable near-infrared conjugated-polymer nanoparticles for dually photoactive tumor inhibition," Advanced Materials, vol. 29, no. 31, article 1700487, 2017.

[38] Z. Guo, Y. Zou, H. He et al., "Bifunctional platinated nanoparticles for photoinduced tumor ablation," Advanced Materials, vol. 28 , no. 46 , pp. 10155-10164, 2016.
[39] S. Ye, J. Rao, S. Qiu et al., "Rational design of conjugated photosensitizers with controllable photoconversion for dually cooperative phototherapy," Advanced Materials, vol. 30, no. 29, article 1801216, 2018.

[40] G.-J. Zhou, W.-Y. Wong, D. Cui, and C. Ye, "Large opticallimiting response in some solution-processable polyplatinaynes," Chemistry of Materials, vol. 17, no. 20, pp. 5209-5217, 2005.

[41] W.-Y. Wong, X. Z. Wang, Z. He et al., "Metallated conjugated polymers as a new avenue towards high-efficiency polymer solar cells," Nature Materials, vol. 6, no. 7, pp. 521-527, 2007.

[42] W.-Y. Wong and C.-L. Ho, "Organometallic photovoltaics: a new and versatile approach for harvesting solar energy using conjugated polymetallaynes," Accounts of Chemical Research, vol. 43, no. 9, pp. 1246-1256, 2010.

[43] A. Haque, R. A. Al-Balushi, I. J. Al-Busaidi, M. S. Khan, and P. R. Raithby, "Rise of conjugated poly-ynes and poly(metalla-ynes): from design through synthesis to structureproperty relationships and applications," Chemical Reviews, vol. 118, no. 18, pp. 8474-8597, 2018.

[44] G. Li, W. Hu, M. Zhao et al., "Rational design of near-infrared platinum(ii)-acetylide conjugated polymers for photoacoustic imaging-guided synergistic phototherapy under $808 \mathrm{~nm}$ irradiation," Journal of Materials Chemistry B, vol. 8, no. 33, pp. 7356-7364, 2020.

[45] T. Huang, M. Zhao, Q. Yu et al., "De novo design of polymeric carrier to photothermally release singlet oxygen for hypoxic tumor treatment," Research, vol. 2019, article 9269081, pp. 1-11, 2019.

[46] W. Lv, H. Xia, K. Y. Zhang et al., "Photothermal-triggered release of singlet oxygen from an endoperoxide-containing polymeric carrier for killing cancer cells," Materials Horizons, vol. 4, no. 6, pp. 1185-1189, 2017.

[47] I. S. Turan, D. Yildiz, A. Turksoy, G. Gunaydin, and E. U. Akkaya, "A bifunctional photosensitizer for enhanced fractional photodynamic therapy: singlet oxygen generation in the presence and absence of light," Angewandte International Edition Chemie, vol. 55, no. 8, pp. 2875-2878, 2016.

[48] Y. Yuan, N. Zhang, W. Tao, X. Cao, and Y. He, "Fatty acids as phase change materials: a review," Renewable and Sustainable Energy Reviews, vol. 29, pp. 482-498, 2014.

[49] G. Qing, X. Zhao, N. Gong et al., "Thermo-responsive triplefunction nanotransporter for efficient chemo-photothermal therapy of multidrug-resistant bacterial infection," Nature Communications, vol. 10, no. 1, article 4336, 2019.

[50] M. M. Rahman, M. Ueda, T. Hirose, and Y. Ito, "Spontaneous formation of gating lipid domain in uniform-size peptide vesicles for controlled release," Journal of the American Chemical Society, vol. 140, no. 51, pp. 17956-17961, 2018.

[51] J. Xue, C. Zhu, J. Li, H. Li, and Y. Xia, "Integration of phasechange materials with electrospun fibers for promoting neurite outgrowth under controlled release," Advanced Functional Materials, vol. 28, no. 15, article 1705563, 2018.

[52] H. He, S. Ji, Y. He et al., "Photoconversion-tunable fluorophore vesicles for wavelength-dependent photoinduced cancer therapy," Advanced Functional Materials, vol. 29, no. 19, article 1606690, 2017.

[53] H.-S. Peng and D. T. Chiu, "Soft fluorescent nanomaterials for biological and biomedical imaging," Chemical Society Reviews, vol. 44, no. 14, pp. 4699-4722, 2015.

[54] K. Haskins-Glusac, M. R. Pinto, C. Y. Tan, and K. S. Schanze, "Luminescence quenching of a phosphorescent conjugated 
polyelectrolyte," Journal of the American Chemical Society, vol. 126, no. 45, pp. 14964-14971, 2004.

[55] W.-Y. Wong, G.-L. Lu, K.-H. Choi, and J.-X. Shi, "Synthesis and electronic properties of new photoluminescent platinum-containing polyynes with 9,9-dihexylfluorene and 9-butylcarbazole units," Macromolecules, vol. 35, no. 9, pp. 3506-3513, 2002.

[56] E. Glimsdal, M. Carlsson, T. Kindahl, M. Lindgren, C. Lopes, and B. Eliasson, "Luminescence, singlet oxygen production, and optical power limiting of some diacetylide platinum(II) diphosphine complexes," The Journal of Physical Chemistry A, vol. 114, no. 10, pp. 3431-3442, 2010.

[57] T. Cardolaccia, Y. Li, and K. Schanze, "Phosphorescent platinum acetylide organogelators," Journal of the American Chemical Society, vol. 130, no. 8, pp. 2535-2545, 2008.

[58] W. Yang, A. Karatay, J. Zhao et al., "Near-IR broadbandabsorbing trans-bisphosphine $\mathrm{Pt}(\mathrm{II})$ bisacetylide complexes: preparation and study of the photophysics," Inorganic Chemistry, vol. 54, no. 15, pp. 7492-7505, 2015.

[59] B. Guo, Z. Sheng, D. Hu et al., "Molecular engineering of conjugated polymers for biocompatible organic nanoparticles with highly efficient photoacoustic and photothermal performance in cancer theranostics," ACS Nano, vol. 11, no. 10, pp. 10124-10134, 2017.

[60] Q. Zou, M. Abbas, L. Zhao, S. Li, G. Shen, and X. Yan, "Biological photothermal nanodots based on self-assembly of peptideporphyrin conjugates for antitumor therapy," Journal of the American Chemical Society, vol. 139, no. 5, pp. 1921-1927, 2017.

[61] Y. Xu, M. Zhao, L. Zou et al., "Highly stable and multifunctional aza-bodipy-based phototherapeutic agent for anticancer treatment," ACS Applied Materials \& Interfaces, vol. 10, no. 51, pp. 44324-44335, 2018.

[62] J. Li, X. Zhen, Y. Lyu, Y. Jiang, J. Huang, and K. Pu, “Cell membrane coated semiconducting polymer nanoparticles for enhanced multimodal cancer phototheranostics," ACS Nano, vol. 12, no. 8, pp. 8520-8530, 2018.

[63] A. Zhu, K. Miao, Y. Deng et al., "Dually pH/reduction-responsive vesicles for ultrahigh-contrast fluorescence imaging and thermo-chemotherapy-synergized tumor ablation," ACS Nano, vol. 9, no. 8, pp. 7874-7885, 2015.

[64] G. Li, D. Li, L. Zhang, J. Zhai, and E. Wang, "One-step synthesis of folic acid protected gold nanoparticles and their receptor-mediated intracellular uptake," Chemistry A European Journal, vol. 15, no. 38, pp. 9868-9873, 2009.

[65] D. Zheng, Y. Chen, S. Ai et al., "Tandem molecular selfassembly selectively inhibits lung cancer cells by inducing endoplasmic reticulum stress," Research, vol. 2019, article 4803624, pp. 1-11, 2019. 